of Clara cells decreases, and if the bond of the $C C$ with the epithelial layer is weakly expressed upon cooling, Clark cells are in contact with the epithelium under the action of DKV. DKV has a normalizing effect on LPO reactions. In lung tissue, this results in a $16 \%$ decrease in diene conjugate values, hydroperoxides by $10 \%$ and vitamin E growth by $15 \%$. In peripheral blood, hydropericidia are significantly reduced by $20 \%$, MDA by $40 \%$, vitamin E increases by $13 \%$ and ceruloplasmin by $15 \%$.

With the aging of the body, the regeneration potential is reduced as a result of both a decrease in the number of stem cells, as well as the accumulation of metabolic products, which have a damaging effect on cells. With age, there are changes in connective tissue interactions in the epithelial lining of the respiratory tract, which leads to the development of involutive processes [1]. The effect of low temperature in older animals changes the level of proliferative processes more significantly, whichcandistortthedirection ofdifferentiation intheepithelium oftherespiratorysystem [3].Theresultsofthestudyindicate that the negative effect of low temperatures on the body can be reduced by using an antioxidant drug - dihydroquercetin.

\title{
References:
}

1. Pavlov, A.V. Histophysiology of the tracheal epithelium in rats in postnatal ontogenesis / A.V. Pavlov, L.I. Esev // Morphology. 2014. - T.146, №6. - P. 80-86.

2. Teselkin, Yu.O. Antioxidant effect of dihydroquercetin / Yu.O. Teselkin, B.A. Shambilova, I.V. Babenkova, G.I. Klebanov, N.A. Tyukavkin // Biophysics. - 1996. - T. 41, №3. - P. 620-624.

3. Celuyko, S.S. Modern views on the proliferation and differentiation of stem cells of the respiratory system in normal and cold exposure / S.S. Tseluyko, N.P. Krasavina, D.A. Semenov // Bulletin of the physiology and pathology of respiration. - 2012. - №45. P. 98-103.

UDK 617-089:616-08-031.81 DOI 10.22448/AMJ.2017.3.140-141

\section{POSSIBILITIES OF THERAPY OPTIMIZATION OF PATIENTS WITH SUPERFICIAL BURNING WOUNDS}

\author{
O.S. Olifirova, A.A. Kozka, E.A. Volosenkova
}

Amur State Medical Academy,

Gorkogo street, 95, Blagoveshchensk, 675000, tel. 8-(4162)-31-90-20, e-mail: agma@nm.ru

Abstract The analysis of treatment of 88 patients with burns of the I-II degrees (MKB-10) was carried out. Antioxidants (("Lavitol cosmetic", BAA "Lavitol-B") and hyperbaric oxygenation were applied in the complex treatment of patients of the main group (44) until the wound was completely epithelialized. The comparative assessment of results was carried out in a group of patients (44) who received traditional treatment. Antioxidants and hyperbaric oxygenation favorably affect the course of the wound process.The terms of spontaneous epithelization of the wound and inpatient treatment in patients of the main group decreased due to stimulation of the wound process.

Key words: superficial burns, dihydroquercetin, hyperbaric oxygenation, wound process.

Topicality. Burns therapy presents a global, social and economic problem in combustiology and surgery. It is known that among victims patients with superficial and border-line burns prevail making up $80 \%$ [1]. Such injuries do not lead to the development of a burn disease, they need the effective conservative treatment which can stimulate regenerative processes in a zone of the burn. According to modern literature hyperbaric oxygenation stirs up phagocytic activity of cells reparation and rate of their passing into the wound, stimulates action of antibacterial therapy [2]. Besides, in pathogenesis of a process developing in the wound great attention is paid to the active part of uncontrolled formation of free radicals in cells which further deceleration of reparation processes [3]. Application of antioxidants is pathogenetically substantiated. Because dihydroquercetin is a strong exogenous antioxidant having capillary protective, anti-inflammatory and moisture keeping effects then the probability of influence on the processes of LPO (lipid peroxidation) is high that intensifies the fibroblast reaction [4]. Objective: to assess the results of a complex application of hyperbaric oxygenation and dihydroquercetin ("Lavitol cosmetic", BAA "Lavitol-B") in the treatment of patients with superficial burns.

Materials and methods: the analysis of therapy results of 88 patients with burns of the I-II degrees (MKB-10) was conducted. Among them there were 49 men $(55,7 \%)$ and 39 women $(44,3 \%)$ at the age of 20-71 years. Etiology of thermal injuries: scalds - in $50(56,8 \%)$, flame burns - in $25(28,4 \%)$, contact burns - in $11(12,5 \%)$, scalds - in $2(2,3 \%)$ cases. Burn shock of the I-II degrees was observed in 6 patients with a burn area of $15 \%$ and $18 \%$. A burn disease was developing from 14 days till 2 months. On the average the area of thermal injuries made up 630,8 $\pm 6,7 \mathrm{~cm} 2$.

The main group (MG) included 44 patients receiving $\mathrm{HBO}$ and antioxidant therapy with bioflavonoid dihydroquercetin ("Lavitol cosmetic", BAA "Lavitol - B"). Therapy methods of patients of the main group were the following: after thorough cleansing of a burning wound, removing exfoliated epidermis and treatment with antiseptic ( $0,5 \%$ solution of chlorhexidine bigluconate) dressings with the powder of dihydroquercetin "Lavitol cosmetic" were performed in a day during 10-20 days. It was applied to the wound with the presence of microbes not more than $103-4$ b.m. per $1 \mathrm{~cm} 2$ in the layer of 1-2 mm. Atraumatic covering "Parapran" was applied over. At the same time hyperbaric oxygenation of 1,5-1,8 atmospheres during 40 minutes was conducted daily for 10 days. Besides, peroral intake of biologically active additive "Lavitol - B" was administered ( 1 capsule twice a day during meals daily for 15 days). Biologically active additive "Lavitol - B" contains natural antioxidant dihydroquercetin and polysaccharide arabinogalactan (1:3). Patients of the main group and the control group are similar in sex, age, degree and burns area.

Complex of therapy methods for patients from both groups included antibacterial and symptomatic treatment. 
The results were analyzed according to indices of developing a burning disease, cytological examination, lipid peroxidation study and antioxidant defense (vitamin "E", ceruloplasmin), pro-inflammatory interleukins (IL-1 $\beta$, IL-6, IL-8, TNF- $\alpha$ ), ) on the first and 10-th day of therapy. The degree of differences was considered to be significant when $p<0,05$.

Results: Due to more active processes developing in the wound earlier terms of forming marginal ( $7,6 \pm 1,6$ days) and complete epithelization of wounds $(28,2 \pm 3,3$ days), scarring $(31,3 \pm 2,4$ days) $(p<0,05)$ were determined for certain in patients of the main group in comparison with patients of the control group $(10,7 \pm 1,4$ days, $20,9 \pm 3,8$ days, $29,9 \pm 2,3$ days, $39,7 \pm 3,2$ days accordingly). Duration of the temperature reaction is $11,9 \pm 0,6$ days and that of the painful syndrome in the main group made up 13,4 $\pm 0,7$ days. These indices in the control group made up 16,6 \pm 0,7 days and $19,9 \pm 1,5$ days accordingly $(p<0,05)$.

Cellular composition of the wounds on the first day of therapy did not significantly differ in patients of both groups. In the 21-st day of therapy regenerative and regenerative - inflammatory types of cytograms were determined in patients of the main group while inflammatory and regenerative type of cytograms remained in patients of the control group.

Patients with superficial burns in both groups had excessive and lingering hyperproduction of pro-inflammatory interleukins (IL-1 $\beta$, IL-6, IL-8, TNF- $\alpha$ ). Application of the proposed method furthered the decrease of their level and acceleration of reparation processes. By the 10-th day of therapy in patients of the main group having burning wounds the level of IL-1 $\beta$ content in the serum decreased by $45,5 \%$ ( from $132,9[21,5 ; 302]$ to $72,4[8,7 ; 127,6]$ ), IL-6 - 40\% (from 112,5 [51,4; 216,7] to67,5 [13,9; 120,8], IL-8 - 49,3\% (from 230,4 [123,8; 328,5] to $116,7[69,8 ; 254,6]$ ) and TNF- $\alpha-62,8 \%$ (from $43,2[15,04 ; 67]$ to $16,07[6,7 ; 45,8]$ ), and in the control group only $-18,3 \%, 2,9 \%, 3,6 \%, 6,5 \%$, accordingly.

At the beginning of therapy there were not significant differences in indices of lipid peroxidation and AOD in patients of both groups. M.u. indices decreased by $46,8 \%$ and r.c. indices - by $47,9 \%$ in patients of the main group by the tenth day of treatment while m.u. indices decreased by $20,4 \%$ and r.c. indices - by $31,7 \%$ in patients of the control group. The content of AOD components increased significantly in the main group than in the control one. In patients of the main group the content of vitamin "E" increased by $39,1 \%$ and that of ceruloplasmin - by $35,4 \%$ and in the control group - by $8,7 \%$ and - by $3,6 \%$ accordingly.

Conclusion. Application of HBO and antioxidant therapy with dihydroquercetin (Lavitol cosmetic", BAA "Lavitol - B").) activates the process developing in the wound that allows to improve the results of treatment of patients with superficial burns.

\title{
REFERENCES
}

1. Karyakin N.N. Technologies of burns treatment in the conditions of humidity // International journal of applied and fundamental investigations .2015. № 9 (part 3). P. 495-499.

2. Bagaev V.G., Sergeeva V.V., Bobrova A.A. etc. Hyperbaric oxygenation in the complex therapy of wounds in children // Wounds and wound infections. 2014. V.1, № 2. P. 31-37.

3. Lutsevich O.E., Tamrazova O.V., Shikunova A.U. etc. Modern view on pathology and treatment of purulent wounds // Surgery. 2011. № 5. P. 72-77.

4. Lavitol cosmetic. URL: https://www.ametis.ru/production/lavitol-cosmetics (date of address 15.07.2017).

AUTHORS: Olifirova Olga Stepanovna - Doc. Med. Sc., Head of the department of surgical diseases FRAE, tel. 89145544652, mail: olif.oc@mail.ru Kozka Aleksandra Aleksandrovna - post-graduate of the department of surgical diseases FRAE, tel.89638488407, mail: kozka.a.89@mail.ru Volosenkova Elena Alekseevna - assistant of the department of foreign languages

UDC 616.24-001-616.248 DOI 10.22448/AMJ.2017.3.141-142

\section{FEATURES OF BRONCHIAL ASTHMA CLINICAL COURSE IN CONJUNCTION WITH BRONCHIECTASIS}

\author{
Prikhodko O.B., Kostrova I.V., Pogrebnaya M.V., Goryacheva S.A., Smorodina E.I.
}

\author{
Amur state medical academy, Blagoveshchensk, Russian Federation
}

\begin{abstract}
International data indicate an increase in the prevalence of bronchiectasis in recent years. In connection with the expansion of computer diagnostics abilities and an increase in the detectability of bronchiectasis in various diseases, interest in this problem has greatly increased. Presenting a clinical case of comorbid patient observation with the presence of steroid-dependent bronchial asthma and COPD, the so-called cross-over syndrome, in combination with cystic hypoplasia and bronchiectasis.
\end{abstract}

Key words: bronchiectasis, bronchial obstruction, bronchial asthma

International data are showing an increase in the prevalence of bronchiectasis in recent years. Bronchoectasis (BE) among other lung diseases are taking part from $10 \%$ to $30 \%$. With fluorography of chest organs, it's detected in 1-2 of 1000 people, and in the survey with the use of bronchography - in $0.5 \%$ of the population. According to pathoanatomical studies, bronchiectasis occurs in $2-4 \%$ of the adult population. Bronchiectasis in $2 / 3$ of the patients was noted before the age of 20 years. At the age of 10 years, bronchiectasis is equally common in boys and girls, but among adults, men suffer more often than women 1.5-3.0 times [1,2,3].

Material and methods. In connection with the expansion of the possibilities of computer diagnostics (use of high-resolution and spiral computed tomography) and an increase in the detectability of bronchiectasis in various diseases, interest in this problem has visibly increased. In Europe F.C. Ringshausen et al. reported an increase in the number of 